

\title{
Salud mental como predictor de egreso en pacientes con tuberculosis atendidos en centros de alta incidencia. Lima, Perú. 2015-2017
}

\author{
María P. Requena-Herrera', Enrique O. Bedoya-Ismodes', José Aymar Vitorino' y Alonso Soto ${ }^{1,2}$
}

\section{State of mental health as a predictor for the outcome in patients with tuberculosis in health centers with high incidence. Lima, Peru. 2015-2017}

Background: Mental health problems in people with tuberculosis (TB) have been associated with negative outcomes. Some health care centers in Peru use an evaluation of mental health status proposed by the Dirección de Prevención y Control de Tuberculosis (DPCTB). Aim: To evaluate the association between the mental health evaluation indicators at the beginning of treatment and the outcome of people treated with TB. Methods: A retrospective cohort study in three health care centers from the district San Juan de Miraflores. The outcome was considered positive (cured) and negative (failure, dropout, death or change of treatment scheme). Results: A significant association was found between the negative outcome and depressive symptoms (RR: 2.39, 95\%CI: 1.19-4.78), substance abuse (RR: 2.58; 95\%CI: 1.31-5.09) and unprotected sexual intercourse $(\mathrm{p}=0.04)$. The multivariate analysis found association with depressive symptoms (RR: $2.19,95 \% \mathrm{CI}$ : 1.10-4.35) and substance abuse (RR: 2.19, 95\%CI: 1.14-4.20). Conclusion: Depressive symptoms and drug consumption were associated with negative outcomes. Intervention studies focusing on these factors should be evaluated to improve treatment success.

Keywords: Tuberculosis; mental health; depression; Yesavage test.

Palabras clave: Tuberculosis; salud mental; negativa al tratamiento; depresión; test de Yesavage.

\section{Introducción}

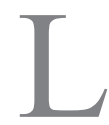

a tuberculosis (TBC) representa uno de los mayores problemas de salud pública con altas tasas de morbi-mortalidad en el mundo y en América Latina. La TBC es una de las principales causas de muerte a nivel global $^{1,2}$, siendo responsable de 1,3 millones de muertes prevenibles en el año $2017^{1}$.

Perú es considerado uno de los países con mayor incidencia de TBC en América con 27 mil casos nuevos de enfermedad activa y 17 mil casos nuevos de TBC pulmonar frotis positivo cada año, siendo Lima, el departamento con mayor concentración de casos en el país $(60 \%)$ y el tercer departamento con más alta incidencia ${ }^{2-4}$. La Dirección de Prevención y Control de Tuberculosis (DPCTB) sigue las recomendaciones de la estrategia Fin de la TBC propuestas por la Organización Mundial de la Salud (OMS) como el uso del Directly Observed Treatment, Short-course (DOTS), entre otros, a fin de disminuir tasas de morbi-mortalidad mediante la detección temprana de casos, adherencia y éxito del tratamiento $^{5-8}$. A pesar de ello, en los últimos años se han incrementado las cepas resistentes, reportándose alrededor de 1.500 casos con TBC multidrogo resis- tente (TBC-MDR) y 100 casos de TBC extensamente resistente (TBC-XDR) por año, los cuales se encuentran predominantemente en Lima.

La condición de egreso luego del tratamiento contra la TBC puede ser catalogada como curado, fracaso, abandono, fallecido y cambio de esquema. Estas cuatro últimas categorías representan resultados considerados no favorables. Entre ellos, el más estudiado es el abandono, el que disminuye la eficiencia de la terapia y conlleva al desarrollo de TBC-MDR ${ }^{1}$. El Perú tiene una tasa de abandono que para el año 2012 llegó a 5\% ${ }^{4}$. Entre los factores relacionados a la falla del tratamiento se han reportado las reacciones adversas a los medicamentos, consumo de alcohol, consumo de drogas y co-infección con virus de inmunodeficiencia humana $(\mathrm{VIH})^{9-12}$. Además, los problemas de salud mental son factores influyentes en la finalización o abandono del tratamiento ${ }^{13-15}$. Desórdenes mentales como depresión y ansiedad son prevalentes en personas con $\mathrm{TBC}^{16,18}$. A pesar de ello, en las guías de TBC, tanto nacionales como internacionales, no se enfatiza lo suficiente el componente de salud mental, ni se incluyen protocolos para el manejo psicológico ${ }^{6,7}$. En el país, la DPCTB propone una evaluación psicológica utilizada en algunos centros de salud (CCSS), que incluye
'Escuela de Medicina, Universidad Peruana de Ciencias Aplicadas, Lima, Perú.

2Departamento de Medicina, Hospital Nacional Hipólito Unanue, Lima, Perú.

Los autores declaran no tener conflicto de interés en la publicación del artículo. Estudio autofinanciado.

Recibido: 20 de enero de 2019 Aceptado: 10 de octubre de 2019

Correspondencia a: Enrique Oswaldo Bedoya Ismodes enri1093@gmail.com 
entre sus componentes un test rápido de funciones mentales, un test rápido de depresión, tamizaje de conductas de riesgo, conducta sexual de riesgo, conciencia de la enfermedad (si conoce y acepta el diagnóstico), grado de adherencia (si toma la medicación correctamente y de manera autónoma), si dispone de informes de evaluación, y el tipo de estancia en la clínica (si el paciente se encuentra hospitalizado o si recibe tratamiento ambulatorio) (Anexo 1). Aunque su objetivo es predecir si hay riesgo de abandono al inicio del tratamiento, incluye también variables que son medidas durante y al término del tratamiento, lo que limita su validez del punto de vista conceptual y práctico. En el Perú, un estudio realizado por Anduaga y cols., en el distrito de Chorrillos, utilizó el puntaje total de esta evaluación psicológica y encontró que las personas con un valor de puntaje mayor o igual a

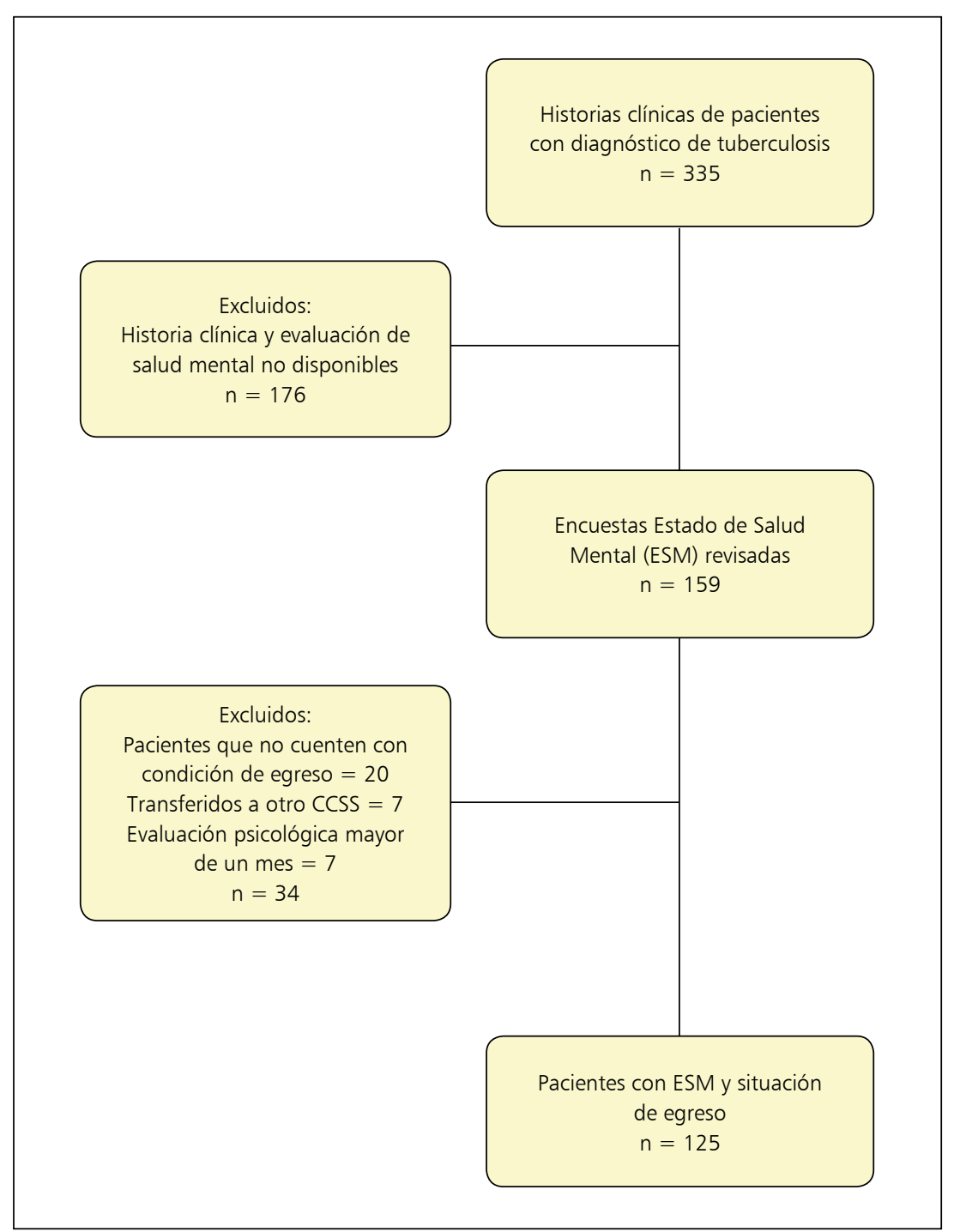

Figura 1. Flujograma.
18 , tenían mayor probabilidad de abandonar el tratamiento $(\mathrm{OR}=21,4)^{19}$. Esta evaluación actualmente es utilizada de manera rutinaria en los CCSS del distrito de San Juan de Miraflores. Sin embargo, no ha sido validada ni sometida a un análisis que estudie la asociación de sus componentes individuales con los desenlaces del tratamiento. Por ello, el propósito de esta investigación es determinar la asociación de las variables incluidas en la evaluación psicológica de la DPCTB al inicio del tratamiento con la condición de egreso en personas que iniciaron el tratamiento antituberculoso en algunos de los CCSS en San Juan de Miraflores, distrito de la ciudad de Lima, Perú.

\section{Materiales y Métodos}

\section{Diseño de estudio}

Estudio analítico de cohorte histórica. Se revisaron las evaluaciones psicológicas (instrumento) de las historias clínicas en tres CCSS de San Juan de Miraflores (Trébol Azul, Manuel Barreto y Leonor Saavedra) en el período de noviembre de 2017 a febrero de 2018.

\section{Población y tamaño de muestra}

Se incluyó en el estudio a todas las personas que fueron diagnosticadas con TBC en los CCSS Trébol Azul, Leonor Saavedra y Manuel Barreto, entre el 1 de enero de 2015 y el 31 de diciembre de 2017, y culminaron el tratamiento hasta 1 de febrero de 2018. Al revisar los registros clínicos se excluyeron a las personas que no contaban con una historia clínica disponible o tenían una evaluación incompleta. Además, se revisaron datos de filiación, fechas de ingreso y la situación de egreso en los libros de registro de cada centro de salud y se excluyó a quienes tenían una evaluación realizada luego de más de un mes de iniciado el tratamiento y a quienes no contaban con una condición de egreso definida (Figura 1).

Se incluyó la totalidad de los registros clínicos en los CCSS, por lo cual no corresponde estrictamente realizar un tamaño muestral. Sin embargo, a efectos referenciales, se calculó el tamaño muestral en el programa EPIDAT 4.2 para un estudio de cohorte. Se consideró una incidencia de $45 \%$ de desenlace no favorable en personas con algún nivel de depresión y una incidencia de $18 \%$ de desenlace no favorable en personas sin depresión ${ }^{20}$, con una razón de tamaño muestral de 1 a 2 , una potencia de $80 \%$ y un nivel de confianza de $95 \%$. Se obtuvo un tamaño muestral referencial de 103 personas.

\section{Definiciones operacionales de las variables principales}

Se consideraron características generales como edad, sexo, estado civil y grado de instrucción. Se agrupó el estado civil en dos categorías; con pareja a los casados 
y convivientes y sin pareja a los solteros, viudos o divorciados. El grado de instrucción se categorizó en: primaria (completa e incompleta), secundaria (completa e incompleta) y superior (completa e incompleta y grados técnicos). También se consideró la localización de TBC en dos categorías, pulmonar y extra-pulmonar; y la sensibilidad al tratamiento antituberculoso, considerando sensible a los fármacos de primera línea y MDR a los resistentes a rifampicina e isoniacida ${ }^{7}$. Por último, se reportó la co-infección con VIH.

Se consideró como herramienta de estudio a la evaluación psicológica de la DPCTB realizada al momento del ingreso del paciente al tratamiento, utilizada por los establecimientos de DIRIS-II LIMA SUR, compuesta por los siguientes ítems:

- Test rápido de funciones mentales (MAJOI): Incluye la evaluación de memoria, afecto, juicio, orientación e intelecto. Según el puntaje de la prueba se categoriza en normal ( $<5$ puntos), deterioro leve ( 6 a 9 puntos) y deterioro profundo $(>10 \text { puntos })^{21}$.

- Test rápido de depresión de Yesavage abreviado ${ }^{22}$ : Se obtiene un puntaje a partir de 15 preguntas. Se categoriza en normal ( $<5$ puntos), sugerente de depresión leve (6 a 9 puntos) y sugerente de depresión profunda (>10 puntos).

- Evaluación de conductas de riesgo: Según la frecuencia del consumo de alcohol, tabaco y drogas. Para cada ítem se considera el puntaje de 3 en consumo frecuente (>3 veces por semana), 2 si es de vez en cuando (1- 3 veces por semana) y 1 si no consume. Se obtiene un riesgo acumulado que resulta de la suma de los tres ítems. Se consideró sin riesgo al puntaje menor o igual a 3 y con riesgo al puntaje mayor de 3 .

- Evaluación de conducta sexual de riesgo: Incluye las relaciones sexuales con dos a más parejas al momento de la evaluación, sin protección de barrera y/o con el mismo sexo. Si alguna de las variables es positiva se considera con riesgo.

- Evaluación de conciencia de enfermedad: Se categoriza en buena si conoce y acepta el diagnóstico y los síntomas de la enfermedad, y deficitaria si tiene conocimiento parcial o nulo de la enfermedad.

A partir de ello, se categorizó la variable dependiente en favorable (curado) y no favorable (fracaso, abandono, fallecido y cambio de esquema), tomando en cuenta las definiciones en la norma técnica del MINSA ${ }^{6}$.

\section{Análisis de datos}

La información obtenida por medio de la recolección de datos de los registros clínicos fue ingresada a una base, mediante doble digitación, en Microsoft Excel 2010 y se registró de acuerdo a la categoría de cada variable. En el análisis descriptivo, las variables numéricas se presen- taron como mediana y rango intercuartil. Las variables categóricas se presentaron en frecuencias y porcentajes. Para el análisis bivariado se utilizó la prueba de U de Mann-Whitney en las variables numéricas. En cuanto a las variables categóricas, se utilizaron las pruebas de $\chi^{2}$ y prueba exacta de Fisher, de acuerdo a los valores esperados. El análisis multivariado se realizó en forma exploratoria utilizando un modelo de regresión de Poisson, con varianzas robustas, incluyendo aquellas variables con valores de p 0,10 en el análisis bivariado. Todos los datos se analizaron utilizando el programa STATA v 14.0, considerando significativo un valor de p 0,05.

\section{Aspectos éticos}

El protocolo fue aprobado por el Comité de Ética de la Universidad Peruana de Ciencias Aplicadas y las autoridades de los CCSS estudiados. El estudio se limitó a la revisión de historias clínicas por lo que no hubo contacto con directo con la población. Cada historia clínica incluida en la base de datos fue nombrada mediante un código, por lo que las personas permanecieron anónimas y no se vio afectada la integridad de las mismos.

\section{Resultados}

Se revisaron 159 historias clínicas que contaban con la evaluación psicológica DPCTB. Se descartaron 20 personas que no contaban con condición de egreso, 7 que fueron transferidos a otro CS y 7 que tenían una evaluación psicológica mayor a un mes del diagnóstico de TBC (Figura 1), quedando 125 registros para el análisis de personas atendidas en: Trébol Azul (41,6\%), Manuel Barreto $(33,6 \%)$ y Leonor Saavedra $(27,8 \%)$ entre los años 2015 y 2017 (Tabla 1).

La mediana de edad fue de 28 años y el género predominante, el masculino $(59,2 \%)$. La mayoría de la población registró ser casados o convivientes $(72,0 \%)$. Contaban con un grado de instrucción predominantemente de secundaria $(55,2 \%)$.

Se presentó una prevalencia de co-infección VIH de 7,2\%. La localización principal de la enfermedad tuberculosa fue pulmonar $(82,4 \%)$ siendo la mayor parte sensible al tratamiento de primera línea $(80,8 \%)$. Respecto a la situación de egreso, la mayoría de personas fueron curados $(78,4 \%)$; es decir, con una condición de egreso favorable (Tabla 1).

En el análisis bivariado (Tabla 2), se encontró una asociación significativa entre la condición de egreso no favorable y la presencia de sintomatología depresiva, consumo de sustancias de riesgo (alcohol, tabaco, drogas y riesgo acumulado) y las relaciones sexuales sin protección. También se encontró que entre las personas co-infectadas con VIH, un tercio presentó un egreso 
Tabla 1. Características de una cohorte de pacientes con tuberculosis atendidos en centros de salud de atención primaria en Lima, Perú. 2015-2017 $(n=125)$

\begin{tabular}{lcc}
\hline Características & $\mathbf{n}(\mathbf{\%})$ \\
\hline Mediana de edad (RIC) & $28(20-43)$ \\
\hline Sexo & & \\
$\quad$ Mujer & 51 & $(40,8 \%)$ \\
Hombre & 74 & $(59,2 \%)$ \\
\hline Estado civil & & \\
Sin pareja & 35 & $(28,0 \%)$ \\
Con pareja & 90 & $(72,0 \%)$ \\
Grado de instrucción & & \\
Primaria & 27 & $(21,6 \%)$ \\
Secundaria & 69 & $(55,2 \%)$ \\
Superior & 29 & $(23,2 \%)$
\end{tabular}

Co-infección con VIH

$\begin{array}{lrr}\text { Sí } & 9 & (7,2 \%) \\ \text { No } & 116 & (92,8 \%)\end{array}$

Localización de TBC

$\begin{array}{lrl}\text { Pulmonar } & 103 & (82,4 \%) \\ \text { Extra-pulmonar } & 22 & (17,6 \%)\end{array}$

Sensibilidad a el tratamiento
Sensible
$101(80,8 \%)$
MDR
$24(19,2 \%)$

Centro de salud

$\begin{array}{lll}\text { Trébol Azul } & 52 & (41,6 \%) \\ \text { Manuel Barreto } & 42 & (33,6 \%) \\ \text { Leonor Saavedra } & 31 & (27,8 \%)\end{array}$

Año de inicio de tratamiento

$\begin{array}{lll}2015 & 34 & (27,2 \%) \\ 2016 & 60 & (48,0 \%) \\ 2017 & 31 & (24,8 \%)\end{array}$

Condición de egreso

$\begin{array}{lrr}\text { Curado } & 98 & (78,4 \%) \\ \text { Fracaso } & 6 & (4,8 \%) \\ \text { Abandono } & 16 & (12,8 \%) \\ \text { Fallecido } & 1 & (0,8 \%) \\ \text { Cambio de esquema } & 4 & (3,2 \%)\end{array}$

RIC: rango intercuartil. VIH: virus de inmunodeficiencia humana. TBC: tuberculosis. MDR: multidrogo-resistente. Sin pareja $=$ soltero, divorciado, viudo. Con pareja = conviviente, casado. Grado de instrucción: Primaria: completa e incompleta, Secundaria: completa e incompleta, Superior: completo e incompleto y técnico. no favorable, comparado con los no co-infectados que presentaron una tasa menor $(20,6 \%)$. Sin embargo, este factor no fue significativo estadísticamente.

En el análisis multivariado, las variables asociadas significativamente a un desenlace no favorable fueron la presencia de sintomatología depresiva (RR: 2,19; IC:1,104,35) y riesgo de consumo de sustancias (RR:2,19; IC:1,14- 4,20) (Tabla 3).

\section{Discusión}

Los principales determinantes pronósticos de la condición de egreso no favorable de las personas tratadas de TB en los CCSS de atención primaria fueron la sintomatología sugerente de depresión, el consumo de sustancias de riesgo y las relaciones sexuales sin protección. El análisis multivariado, realizado de manera exploratoria, sólo encontró relación con los dos primeros.

Estudios realizados sobre salud mental en personas con TBC concuerdan con la asociación con sintomatología depresiva. Hay estudios sobre la influencia de la depresión en la adherencia al tratamiento antituberculoso, en el que se encontró una asociación estadísticamente significativa; las personas sin depresión tenían $82,1 \%$ de adherencia al tratamiento mientras que los que presentaban depresión, dependiendo de su intensidad (leve, moderada o profunda), presentaban de 17,6 a $76,7 \%$ de falla del tratamiento ${ }^{18}$. Además, en un estudio realizado en Etiopía, se encontró que los pacientes que presentan depresión tienen $3,9 \%$ de probabilidad de presentar un tratamiento con desenlace desfavorable a los 6 meses del tratamiento antituberculoso ${ }^{23}$. Otro estudio, más reciente, demostró que las personas con síntomas sugestivos de episodios de depresión mayor presentaban 3,5 más veces de riesgo de tener un desenlace negativo ${ }^{24}$. Un estudio realizado en Pucallpa encontró una relación significativa entre el nivel de depresión y la mala adherencia al tratamiento ${ }^{25}$. De misma manera, estudios internacionales demuestran que la depresión y ansiedad presentan alta prevalencia respecto al abandono de tratamiento antituberculoso de 43,4 y $42,5 \%$, respectivamente ${ }^{13-18,26}$.

Estudios realizados a nivel nacional e internacional concuerdan con la asociación del consumo de sustancias psicoactivas y el desenlace no favorable ${ }^{11,27-30}$. Culqui y cols., encontraron una asociación significativa entre el abandono de tratamiento antituberculoso y el consumo de sustancias psicoactivas, como el alcohol, tabaco y drogas, presente en $62,7 \%$ de su población ${ }^{28}$. En otro estudio, se encontró que la presencia de consumo de alcohol presenta un incremento de 3,33 veces de abandonar el tratamiento antituberculoso ${ }^{29}$. Chuang y cols., encontraron que la tasa de éxito de tratamiento antituberculoso en pacientes no consumidores de tabaco es de $77 \%$ en comparación a 
Tabla 2. Factores asociados con la condición de egreso no favorable en pacientes con tuberculosis atendidos en centros de atención primaria de Lima, Perú. 2015- 2017. Resultados análisis bivariado

\begin{tabular}{|c|c|c|c|}
\hline Características & $\begin{array}{l}\text { Egreso no favorable }(n=27) \\
n \quad(\%)\end{array}$ & $\begin{array}{c}\text { Egreso favorable }(n=98) \\
n \quad(\%)\end{array}$ & $p$ \\
\hline Mediana de edad (RIC) & $28(20-42)$ & $27,5(20-43)$ & 0,97 \\
\hline Sexo & & & 0,18 \\
\hline Mujer & $8(15,6)$ & $43(84,3)$ & \\
\hline Hombre & $19(25,6)$ & $55(74,3)$ & \\
\hline Estado civil & & & 0,45 \\
\hline Sin pareja & $6(17,1)$ & $29(82,8)$ & \\
\hline Con pareja & $21(23,3)$ & $69(76,6)$ & \\
\hline Grado de instrucción & & & 0,35 \\
\hline Primaria & $8(29,3)$ & $19(70,3)$ & \\
\hline Secundaria & $15(21,7)$ & $54(78,2)$ & \\
\hline Superior & $4(13,7)$ & $25(86,2)$ & \\
\hline Co-infección con VIH & & & 0,30 \\
\hline Sí & $3(33,3)$ & $6(66,6)$ & \\
\hline No & $24(20,6)$ & $92(79,3)$ & \\
\hline Localización de TBC & & & 0,54 \\
\hline Pulmonar & $22(21,3)$ & $81(78,6)$ & \\
\hline Extra-pulmonar & $5(22,7)$ & $17(77,2)$ & \\
\hline Sensibilidad a el tratamiento & & & 0,36 \\
\hline Sensible & $23(22,7)$ & $78(77,2)$ & \\
\hline MDR & $4(16,6)$ & $20(83,3)$ & \\
\hline Centro de salud & & & 0,38 \\
\hline Trébol Azul & $10(19,2)$ & $42(80,7)$ & \\
\hline Manuel Barreto & $12(28,5)$ & $30(71,4)$ & \\
\hline Leonor Saavedra & $5(16,1)$ & $26(53,8)$ & \\
\hline Año de inicio de tratamiento & & & 0,70 \\
\hline 2015 & $8(23,5)$ & $26(76,4)$ & \\
\hline 2016 & $14(23,3)$ & $46(76,6)$ & \\
\hline 2017 & $5(16,1)$ & $26(83,8)$ & \\
\hline \multicolumn{4}{|l|}{ Evaluación psicológica } \\
\hline \multicolumn{4}{|l|}{ Test rápido de funciones mentales: MAJOI } \\
\hline Normal & $4(14,8)$ & $23(85,1)$ & 0,33 \\
\hline Deterioro leve & $17(22,6)$ & $58(77,3)$ & \\
\hline Deterioro severo & $6(26,0)$ & $17(73,9)$ & \\
\hline \multicolumn{4}{|l|}{ Sintomatología depresiva (Test de Yesavage) } \\
\hline Normal & $10(13,7)$ & $63(86,3)$ & 0,01 \\
\hline Depresión leve & $10(31,2)$ & $22(68,7)$ & \\
\hline Depresión severa & $7(35,0)$ & $13(65,0)$ & \\
\hline \multicolumn{4}{|l|}{ Consumo de sustancias de riesgo } \\
\hline Consumo de alcohol & & & $<0,01$ \\
\hline No consume & $11(12,9)$ & $74(87,0)$ & \\
\hline Consume & $24(60,0)$ & $16(40,0)$ & \\
\hline Consumo de tabaco & & & $<0,01$ \\
\hline No consume & $15(15,1)$ & $84(84,8)$ & \\
\hline Consume & $12(46,1)$ & $12(46,1)$ & \\
\hline Consumo de drogas & & & 0,04 \\
\hline No consume & $18(17,8)$ & $83(82,1)$ & \\
\hline Consume & $9(37,5)$ & $15(62,5)$ & \\
\hline Riesgo acumulado de consumo de sustancias & & & $<0,01$ \\
\hline Sin riesgo & $11(13,7)$ & $69(86,2)$ & \\
\hline Con riesgo & $16(35,5)$ & $29(64,4)$ & \\
\hline
\end{tabular}




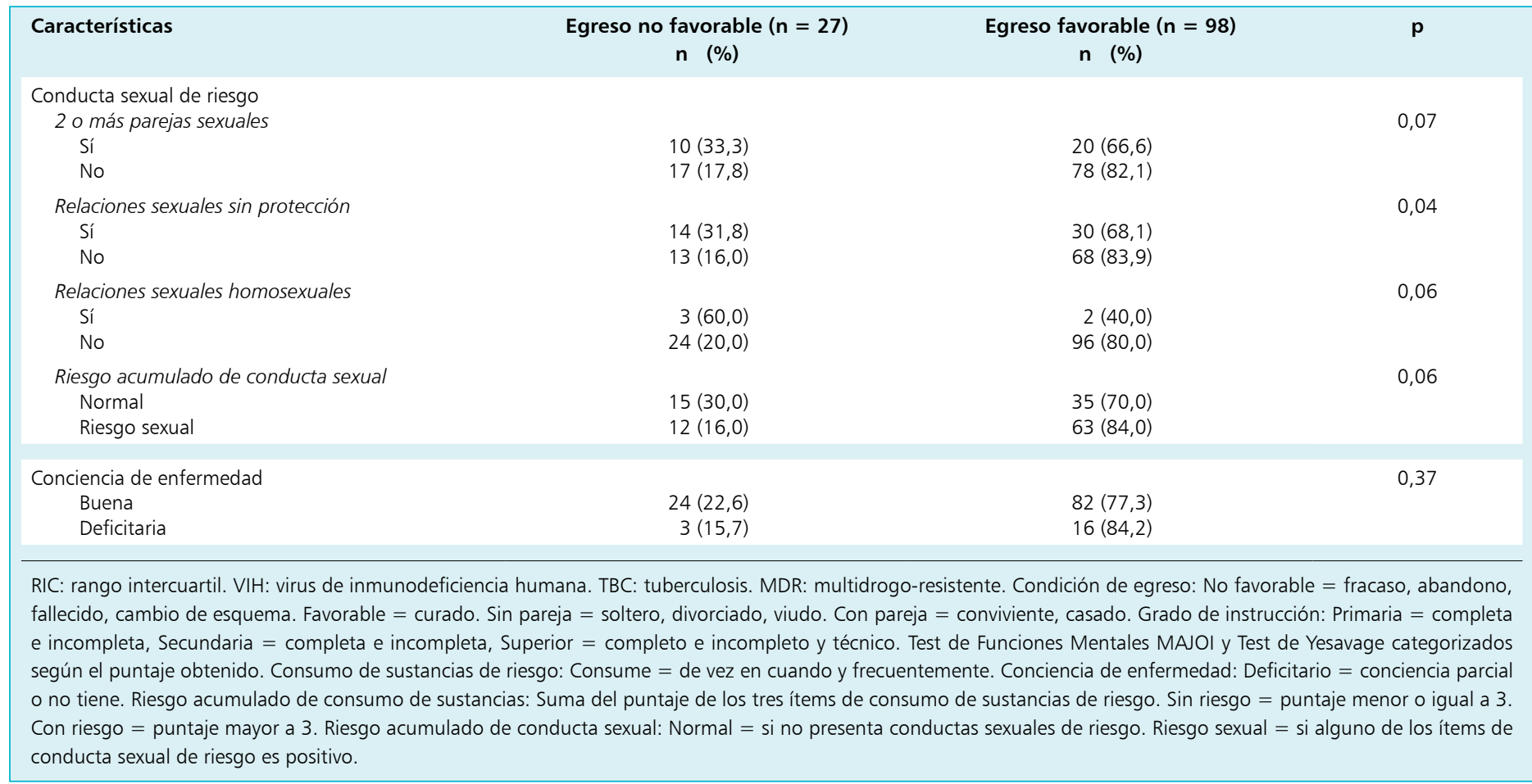

Tabla 3. Factores asociados con la condición de egreso no favorable en pacientes con tuberculosis atendidos en centros de atención primaria de Lima, Perú. 2015- 2017. Modelos crudo y ajustado

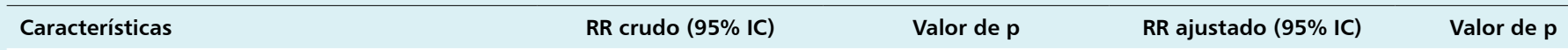

Sintomatología depresiva (Test de Yesavage)

No sugerente de depresión

Ref

Ref

Sugerente de depresión

$2,39(1,19-4,78)$

0,01

$2,19(1,10-4,35)$

0,03

Riesgo acumulado de consumo de sustancias
Sin riesgo
Ref

Ref

Con riesgo

$2.58(1,31-5,09)$

$<0.01$

$2,19(1,14-4,20)$

$<0,01$

Riesgo acumulado de conducta sexual

Sin riesgo

Ref

Ref

Riesgo sexual

$1,88(0,96-3,67)$

0,06

$1,39(0,74-2,60)$

0,30

Ref = Variable de referencia. Sintomatología depresiva: diagnósticos de test de Yesavage categorizados: Depresión = depresión leve y depresión severa. Riesgo acumulado de consumo de sustancias: Suma del puntaje de los tres ítems de consumo de sustancias de riesgo. Sin riesgo = puntaje menor o igual a 3 . Con riesgo = puntaje mayor a 3. Riesgo acumulado de conducta sexual: Normal = si no presenta conductas sexuales de riesgo. Riesgo sexual = si alguno de los ítems de conducta sexual de riesgo es positivo.

los fumadores que es de $67 \%{ }^{30}$. El consumo de tabaco, además de asociarse a un desenlace desfavorable de tratamiento antituberculoso, también afecta la gravedad de la enfermedad, la respuesta bacteriológica y se asocia a aumento de 1,6 veces en las recaídas ${ }^{31}$. Además, se ha demostrado que el cese del consumo de tabaco, reduce la mortalidad asociada a $\mathrm{TBC}^{32}$.
La condición de relación sexual de riesgo no fue significativa en el modelo ajustado del análisis multivariado. Esto se debe probablemente al tamaño de la muestra y a la consecuente limitación del uso de modelos multivariados. No hemos encontrado estudios que relacionen conductas sexuales de riesgo con el abandono del tratamiento o la situación de egreso en personas con TBC. Sin embargo, 
son conductas de riesgo importantes en la infección por $\mathrm{VIH}^{33}$. Un estudio realizado en Colombia, identificó la co-infección TBC-VIH como factor de riesgo para el abandono del tratamiento antituberculoso $(\mathrm{HR}=2,94$, IC95\% 1,60-5,39) $)^{34}$.

En nuestro estudio, el sexo predominante fue el masculino; sin embargo, este no fue un factor asociado al desenlace desfavorable. En contraste, en el estudio de Culqui y cols., se encontró que el sexo masculino tiene 1,62 más veces el riesgo de abandonar el tratamiento ${ }^{28}$. Otro estudio realizado en Perú, encontró un incremento 7,0 veces en el desenlace no favorable comparado con el sexo femenino ${ }^{29}$. En una publicación brasileña se encontró que los hombres presentaban dos veces el riesgo de abandonar el tratamiento a comparación de las mujeres ${ }^{11}$.

El presente estudio demuestra la importancia de valorar la salud mental en la evaluación inicial del paciente con TBC que va a iniciar tratamiento, pues es un importante predictor del resultado del mismo. Aunque se enfatiza mucho la condición de multidrogo-resistencia como posible predictor de malos resultados, en nuestro estudio no fue un predictor significativo, mientras que los problemas de salud mental, particularmente la depresión y consumo de sustancias, incrementaron significativamente el riesgo de malos resultados.

Este es uno de los primeros estudios que evalúan la asociación de los factores de riesgo identificados en la evaluación psicológica que pueden ser importantes predictores de la condición de egreso. Sin embargo, al tratarse de un estudio retrospectivo basado en registros clínicos, presenta limitaciones, en particular con respecto al control del llenado adecuado de las evaluaciones. También se debe tener en cuenta que no todas las historias clínicas cuentan con los datos completos, por lo cual la población de estudio se vio reducida. Por último, el estudio se realizó sólo en los CCSS de la DIRIS-II LIMA SUR, perteneciente al MINSA por lo cual los resultados no son extrapolables a otras poblaciones, como los asegurados por EsSalud, pertenecientes a las Fuerzas Armadas o el sector privado.

Es importante realizar un diagnóstico temprano de la sintomatología sugerente de depresión, el consumo de sustancias de riesgo y las relaciones sexuales sin protección, identificados en nuestro estudio, como factores de riesgo, para realizar intervenciones a fin de evitar un posible desenlace desfavorable. Las intervenciones se deben dirigir a la promoción y prevención de estos factores de riesgo con un trabajo integral a cargo de los diferentes miembros del equipo de salud.

Se debe reevaluar la evaluación psicológica que se utiliza rutinariamente en los CCSS evaluados. Por ello, importante replantear la herramienta, para perfeccionarla y validarla en base a elementos basados en evidencia, como los determinantes significativos que se obtuvieron en nuestro estudio. La sintomatología depresiva fue un factor importante, tanto en la bibliografía revisada como en los resultados del presente estudio; sin embargo, el test de Yesavage utilizado podría no ser el más adecuado, pues es una prueba diseñada para el adulto mayor, por lo cual se podría considerar utilizar una herramienta alternativa para población general ${ }^{21}$. Además, la predicción de desenlaces debe evaluarse al ingreso al tratamiento para identificar factores sobre los que pueda realizarse una intervención temprana. Sin embargo, la herramienta actualmente utilizada toma en cuenta parámetros de seguimiento estrechamente relacionados al desenlace, como la adherencia (no incluida como predictor en nuestro estudio, dado que evaluamos variables al ingreso al tratamiento). El contar con reglas de predicción clínica al ingreso al tratamiento permitirá el desarrollo de intervenciones destinadas a mejorar los desenlaces clínicos relevantes. Al respecto, un ensayo clínico en Lima y provincias, realizó un seguimiento de cinco años en el cual se intervino el aspecto psicosocial. El impacto de las intervenciones no sólo ayudó a las personas a manejar dificultades asociadas y la adherencia al tratamiento antituberculoso; también creó redes sociales y mejora en calidad de vida de las personas y su entorno ${ }^{35}$. Sin embargo, es importante realizar estudios de intervención sobre desenlaces objetivos e importantes para el paciente, en particular la condición de curado.

En conclusión, entre los componentes de la evaluación psicológica utilizada, sólo la sintomatología depresiva y el consumo de sustancias de riesgo se asociaron consistentemente a desenlaces no favorables en pacientes que inician tratamiento de la TBC. En base a estos resultados, la herramienta utilizada como encuesta psicológica en los CCSS estudiados, debería ser cuidadosamente replanteada y validada en base a elementos basados en evidencia. Recomendamos realizar estudios de intervención sobre estos determinantes de un egreso no favorable a fin de mejorar los resultados del tratamiento antituberculoso.

Agradecimientos. A los doctores María Mesta, Alexander Ascarruz y María Félix quienes son consultores locales del programa de Prevención y Control de Tuberculosis en el Centro Materno Infantil Manuel Barreto, Centro de Salud Leonor Saavedra y Centro de Salud Trébol Azul, respectivamente, permitiéndonos el acceso a las instalaciones y el acceso a la información de las historias clínicas.

\section{Resumen}

Introducción: La problemática de salud mental en personas con tuberculosis (TBC) ha sido asociada a desenlaces no favorables. En algunos centros de salud (CCSS) del Perú se utiliza una evaluación del estado de salud mental propuesta por la Dirección de Prevención 
y Control de Tuberculosis, Perú. Objetivos: Evaluar la asociación entre los indicadores de la evaluación de salud mental al inicio del tratamiento y la condición de egreso de personas tratadas con TBC. Material y Métodos: Estudio de cohorte retrospectivo en tres CCSS del distrito de San Juan de Miraflores. La condición de egreso se consideró favorable (curados) y no favorable (fracaso, abandono, fallecidos o cambio de esquema). Resultados: Se encontró una asociación significativa entre la condición de egreso no favorable con la sintomatología depresiva (RR: 2,39;
IC95\%: 1,19-4,78) y el consumo de sustancias de abuso (RR: 2,58; IC95\%: 1,31-5,09) y actividad sexual no protegida $(\mathrm{p}=0,04)$. El análisis multivariado encontró asociación con la sintomatología depresiva (RR: 2,19; IC95\%: 1,10-4,35) y consumo de sustancias de abuso (RR: 2,19; IC95\%: 1,14-4,20). Conclusión: La sintomatología depresiva y el consumo de sustancias de abuso se asociaron a un desenlace desfavorable. Se debe realizar estudios de intervención sobre estos factores a fin de mejorar el éxito del tratamiento.

\section{Referencias bibliográficas}

1.- WHO. Global Tuberculosis Report 2018. Geneva: World Health Organization; 2018. http://apps.who.int/medicinedocs/documents/ s23553en/s23553en.pdf.

2.- Centro Nacional de Epidemiologia, Prevención y Control de Enfermedades. Vigilancia de Tuberculosis. Lima: Centro Nacional de Epidemiologia, Prevención y Control de Enfermedades; 2017 [citado en: 2017 Ago 24]. Disponible en: http://www.dge.gob.pe/portal/ index.php?option $=$ com_content\&view $=$ article $\&$ id $=420 \&$ Itemid $=358$

3.- OPS. La tuberculosis en la Región de las Américas, Informe Regional 2013. Washington, DC: Organización Panamericana de la Salud; 2014 [citado en: 2017 Ago 24]. Disponible en: http://www2.paho.org/hq/index. php?option $=$ com docman\&task $=$ doc view\&Ite mid $=270 \&$ gid $=22954 \&$ lang $=$ es

4.- MINSA. Análisis de la situación epidemiológica de la tuberculosis en el Perú 2015 Lima, Perú: Ministerio de Salud; 2015 [citado en: 2017 Ago 24]. Disponible en: http:// bvs.minsa.gob.pe/local/MINSA/3446.pdf.

5 WHO. The end TB strategy. Geneva: World Health Organization; 2015 [citado en: 2017 Set 18]. Disponible en: http://www.who.int/tb/ End_TB_brochure.pdf?ua $=1$

6.- Perú, Ministerio de Salud Norma Técnica de Salud para la atención integral de las personas afectadas por TB. Resolución Ministerial $\mathrm{N}^{\circ}$ 715-2013/MINSA del 8 de noviembre del 2013. Lima, Perú: MINSA; 2013 [citado en: 2017 Ago 24]. Disponible en: ftp://ftp2.minsa. gob.pe/descargas/dgsp/ESN-tuberculosis/ normaspublicaciones/NTSTBC.pdf.

7.- WHO. Treatment of Tuberculosis: Guidelines, 4 ed. Geneva: World Health Organization; 2010 [citado en: 2017 Ago 24]. Disponible en: http://apps.who.int/iris/ bitstream/10665/44165/1/9789241547833_eng. pdf?ua $=1 \& u a=1$.

8.- Li C T, Chu K H, Reiher B, Kienene T, Chien L Y. Evaluation of health-related quality of life in patients with tuberculosis who completed treatment in Kiribati.
J Int Med Res 2017; 45 (2): 610-20. doi: $10.1177 / 0300060517694491$.

9.- Valenzuela-Jiménez H, Manrique-Hernández E F, Idrovo A J. Association of tuberculosis with multimorbidity and social networks. J Bras Pneumol. 2017; 43 (1): 51-3. doi: 10.1590/ S1806-37562016000000075.

10.- Imtiaz S, Shield K D, Roerecke M, Samokhvalov A V, Lönnroth K, Rehm J. Alcohol consumption as a risk factor for tuberculosis: meta-analyses and burden of disease. Eur Respir J. 2017; 50(1): 1-13. doi: 10.1183/13993003.00216-2017.

11.- de Faria Gomes N M, da Mota Bastos M C, Marins R M, Barbosa A A, Soares L C, de Oliveira Wilken de Abreu A M, et al. Differences between risk factors associated with tuberculosis treatment abandonment and mortality. Pulm Med 2015; 2015: 1-8. doi: 10.1155/2015/546106.

12.- Silva V D D, Mello F C Q, Figueiredo S C A. Estimated rates of recurrence, cure, and treatment abandonment in patients with pulmonary tuberculosis treated with a -four-drug fixed-dose combination regimen at a tertiary health care facility in the city of Rio de Janeiro, Brazil. J Bras Pneumol 2017; 43 (2): 113-20. doi: 10.1590/S180637562016000000204.

13.- Kehbila J, Ekabe C J, Aminde L N, Noubiap J J, Fon P N, Monekosso G L. Prevalence and correlates of depressive symptoms in adult patients with pulmonary tuberculosis in the Southwest Region of Cameroon. Infect Dis Poverty. 2016; 5 (1): 51. doi: 10.1186/s40249016-0145-6.

14.- Kastien-Hilka T, Rosenkranz B, Bennett B, Sinanovic E, Schwenkglenks M. How to evaluate health-related quality of life and its association with medication adherence in pulmonary tuberculosis - designing a prospective observational study in South Africa. Front Pharmacol 2016; 7: 125. doi: 10.3389/ fphar.2016.00125.

15.- Jaber A A, Khan A H, Syed Sulaiman S A, Ahmad N, Anaam M S. Evaluation of healthrelated quality of life among tuberculosis patients in two cities in Yemen. PLoS One
2016; 11 (6): 1-19. doi: https://doi.org/10.1371/ journal.pone. 0156258 .

16.- Duko B. Gebeyehu A, Ayano G. Prevalence and correlates of depression and anxiety among patients with tuberculosis at WolaitaSodo University Hospital and Sodo Health Center, WolaitaSodo, South Ethiopia, Cross sectional study. BMC Psychiatry 2015 15: 214. doi: 10.1186/s12888-015-0598-3.

17.- Salodia U P, Sethi S, Khokhar A. Depression among tuberculosis patients attending a DOTS centre in a rural area of Delhi: A cross-sectional study. Indian J Public Health. 2019; 63 (1): 3943- doi: 10.4103/ijph.IJPH_109_18.

18.- Pachi A, Bratis D, Moussas G, Tselebis A. Psychiatric morbidity and other factors affecting treatment adherence in pulmonary tuberculosis patients. Tuberc Res Treat. 2013: 1-37. doi: 10.1155/2013/489865.

19.- Anduaga-Beramendi A, Maticorena-Quevedo J, Beas R, Chaname-Baca D M, Veramendi M, Wiegering-Rospigliosi A, et al. Factores de riesgo para el abandono del tratamiento de tuberculosis pulmonar sensible en un establecimiento de salud de atención primaria, Lima, Perú. Acta Méd. Peruana 2016; 33 (1): 21-8. http://www.scielo.org.pe/pdf/amp/v33n1/ a05v33n1.pdf.

20.- Morales H. Influencia de la depresión sobre la adherencia al tratamiento en pacientes TB- MDR de la Red Almenara. Revista de la Facultad de Medicina Humana de la Universidad Ricardo Palma. 2015; 15(1): 1622. http://revistas.urp.edu.pe/index.php/RFMH/ article/view/981/887.

21.- Arroyo E, Chamorro J, Castañeda C. Adaptación y validación del test Evaluación Rápida de las Funciones Cognitivas (Gil et al, 1986). Primera versión en castellano con una muestra geriátrica. Psiq Biol 2009; 16 (3): 112 21.

22.- Martínez De La Iglesia J, Onís Vilches C, Dueñas Herrero R, Albert Colomer C, Aguado Taberné C, Luque R. Versión española del cuestionario de Yesavage abreviado (GDS) para el despistaje de depresión en mayores de 65 años: adaptación y validación. MEDIFAM 2002; 12 (10): 620-30. 
23.- Ambaw F, Mayston R, Hanlon C, Medhin G, Alem A. Untreated depression and tuberculosis treatment outcomes, quality of life and disability, Ethiopia. Bull World Health Organ 2018; 96 (4): 243-55. doi:10.2471/ BLT.17.192658.

24.- Ugarte-Gil C, Ruiz P, Zamudio C, Canaza L, Otero L, et al. Association of major depressive episode with negative outcomes of tuberculosis treatment. PLoS ONE 2013; 8 (7): e69514. doi: 10.1371/journal.pone.0069514.

25.- Maldonado A, Lozano L. Influencia de la depresión sobre adherencia al tratamiento en pacientes con tuberculosis multidrogoresistente en el hospital regional de Pucallpaen el período enero de 2014- diciembre de 2015. [Tesis pregrado]. Universidad Nacional de Ucayali; 2016.

26.- Yilmaz A, Dedeli O. Assessment of anxiety, depression, loneliness and stigmatization in patients with tuberculosis. Acta Paul Enferm 2016; 29(5): 549-57. Yilmaz, A., \& Dedeli, O. Assessment of anxiety, depression, loneliness and stigmatization in patients with tuberculosis. Acta Paulista de Enfermagem 2016; 29 (5): 549-57. doi: 10.1590/1982-0194201600076.
27.- Silva M, Pereira J, Costa R R, Dias J A, Guimarães M D C, Leite I C G. Drug addiction and alcoholism as predictors for tuberculosis treatment default in Brazil: a prospective cohort study. Epidemiol Infect 2017; 145 (16): 351624. doi: 10.1017/S0950268817002631.

28.- Culqui D, Munayco C, Grijalva C, Cayla J, Horna- Campos O, Alva K, et al. Factors associated with the Non- completion of conventional anti-tuberculosis treatment in Peru. Arch Bronconeumol 2012; 48 (5): 150155. doi: 10.1016/j.arbres.2011.12.008.

29.- Chavez-Salazar J, Fabian-Pantoja B, Joseph Loza-Delgadillo A, Valladares-Zevallos G. Factores socioeconómicos asociados al abandono del tratamiento de tuberculosis pulmonar sensible en la micro-red de salud ollantay en San Juan de Miraflores, Lima-Perú, 2012-2015. 2017; 17 (4): 12-8. doi: https://doi. org/10.25176/RFMH.v17.n4.1204.

30.- Chuang HC, Su CL, Liu HC, Feng PH, Lee $\mathrm{KY}$, Chuang KJ, et al. Cigarette smoke is a risk factor for severity and treatment outcome in patients with culture-positive tuberculosis. Ther Clin Risk Manag 2015; 11: 1539-44. doi: 10.2147/TCRM.S87218.

31.- Leung CC, Yew WW, Chan CK, Chang KC,
Law WS, Lee SN, et al. Smoking adversely affects treatment response, outcome and relapse in tuberculosis. Eur Respir J 2015; 45: 738-45. doi: 10.1183/09031936.00114214.

32.- Rossato D, Muñoz- Torrico M, Duarte R, Galvão T, Bonini EH, Arbex FF, et al. Risk factor for tuberculosis: diabetes, smoking, alcohol use, and the use of drugs. J Bras Pneumol 2018; 44 (2): 145-52. doi: 10.1590/ s1806-37562017000000443.

33.- WHO. Position Statement: Condoms and HIV prevention. Geneva: World Health Organization; 2019 [citado en: 2019 Mar 20]. Disponible en: http://www.who.int/hiv/ pub/condoms/20090318_position_condoms. pdf?ua $=1$.

34.- Cáceres F d M, Orozco L C. Incidencia y factores asociados al abandono del tratamiento antituberculoso. Biomédica. 2007;27(4):498504. doi: https://doi.org/10.7705/biomedica. v27i4.170.

35.- Acha J, Sweetland A, Guerra D, Chalco K, Castillo H, Palacios E. Psychosocial support groups for patients with multidrug-resistant tuberculosis: five years of experience. Glob Public Health 2007; 2 (4): 404-17. doi: 10.1080/17441690701191610. 